\title{
Charged Rosettes at High and Low Ionic Strengths
}

\author{
Helmut Schiessel \\ Max-Planck-Institut für Polymerforschung, Theory Group, P.O. Box 3148, D-55021 Mainz, Germany \\ Received August 27, 2002
}

\begin{abstract}
The complexation between a semiflexible polyelectrolyte and an oppositely charged macroion leads to a multitude of structures ranging from tight complexes with the chain wrapped around the macroion to open multileafed rosettelike complexes. Rosette structures, expected to occur for short-ranged attractions between the macroion and the chain, have now also been seen in recent Monte Carlo simulations with long-range (unscreened) interactions (Macromol ecules 2002, 35, 5183). The current study provides scaling theories for both cases and shows that rosette structures are indeed quite robust against changes in the ionic strength. However, the transition from the wrapped to the rosette configuration has a dramatically different characteristics: The short-range case leads to a sharp transition into a rosette with large leaves whereas the long-range case occurs in a continuous fashion. We provide the complete diagram of states for both cases.
\end{abstract}

\section{Introduction}

Many biological processes involve the complexation between a charged macroi on and an oppositely charged chain. A prominent example is the complexation of DNA with histone proteins which is the basic step of DNA compaction into chromatin in the cells of animals and plants. ${ }^{1}$ Complexation between macroions and synthetic polymers is also encountered in many technological applications as a means to modify macroion solution behavior; examples are the complexation of polymers with charged colloidal particles ${ }^{2,3}$ and charged micelles. ${ }^{4}$

There is now a large body of theoretical studies on this set of problems, most of them have appeared within the last three years ${ }^{5-16}$ (cf. also earlier related stud$i^{12} s^{17-20}$ ). They vary widely with respect to the methods and the level of approximations used and also with respect to the physical properties assumed for the chain and the macroi on-the latter usually being modeled as a charged sphere. Some studies $5,13,14$ assume the chain to be so highly charged that counterion release is the dominant mechanism for the sphere-chain complexation, whereas most of the other studies assume weakly charged components attracted via standard electrostatics. In some cases the ball -chain complexes interact via a short-range attraction $10,15,16,19$ corresponding to high ionic strengths. ${ }^{8}$ The investigated systems also vary with respect to the chain flexibility: semiflexible chains are considered in refs $5,8-10,14-16$, and 19 whereas the other studies assume flexible polymers. References 15 and 16 were devoted to the diffusion (repositioning) of the complexed sphere along the chain. And, finally, complexes between a chain and several spheres have been considered. ${ }^{12,14}$ The space available here does not allow one to give a detailed account of the different approaches as well as of the phenomenons predicted like overcharging, conformational symmetries of the adsorbed chain etc. A critical survey will be provided in a topical review. ${ }^{21}$

Sphere-chain complexes have also been investigated in several computer simulations. ${ }^{22-32}$ Wallin and Linse studied the effect of chain flexibility, 22 line charge density, ${ }^{23}$ and sphere radius ${ }^{24}$ on the geometry of a complex between a charged sphere and a polyelectrolyte in a Monte Carlo simulation that took counterions explicitly into account; they also considered the case when there are many chains present. ${ }^{25}$ Chodanowski and Stol ${ }^{27}$ investigated the adsorption of a flexible chain on a sphere assuming Debye-Hückel interaction. The case of multisphere adsorption on flexible ${ }^{28}$ and semiflexible chains ${ }^{29}$ was studied by J onsson and Linse. Messina, $\mathrm{Holm}$, and Kremer ${ }^{30,31}$ demonstrated that in the case of strong electrostatic coupling it is even possible that a polyelectrolyte chain forms a complex with a sphere that carries a charge of the same sign-a phenomenon made possible by correlation effects due to neutralizing counterions. Finally, a recent systematic study by Akinchina and Linse ${ }^{32}$ focused again on the role of chain flexibility on the structure of the spherechain complex.

The overall picture emerging from this multitude of theoretical and simulation studies is still not very clear. Partly this has to be attributed to the fact that there are many free parameters determining the properties of the sphere-chain complex, especially the length of the chain, its linear charge density and persistence length, the macroion radius and charge, and the screening length of the salt solution. This makes it difficult to develop a theory that covers the whole range of possible structures. The current study is an attempt to give a scaling theory that allows an approximate treatment of the chain-sphere complex over the whole range of parameters and that especially fills the gaps that were left open by the existing theories and simulations. It allows to identify the few independent scaling parameters in this system and leads to the construction of twodimensional phase diagrams (one for short and one for large screening lengths) that cover the whole range of all the other parameters.

This paper has been induced by a comparison of refs 10 and 32. In the former paper we studied the complexation behavior of a semiflexible chain and a "sticky" sphere. In the context of electrostatics this corresponds to high salt concentrations where the Debye screening length is much shorter than the sphere radius. In that paper, we calculated the zero-temperature configurations modeling the polymer by the wormlike chain. We found two typical structures that occur in this system. If the sphere is sticky enough, i.e., if the chain adsorp- 
tion energy per length is large, the chain wraps around the sphere-as long as there is enough surface available. When the adsorption energy is decreased there is a point at which the bent chain unwraps in a sharp fashion. The new structure that emerges has multiple point contacts between the sphere and the chain and large low-curvature loops connecting them. We called this class of structures the rosette configurations.

On the other hand, the latter work, ref 32, presented a Monte Carlo study of the complexation of a semiflexible charged chain with an oppositely charged ball that carries the same absolute charge as the chain (isoel ectric complex). No small ions were present so that the charged monomers were attracted to the sphere via an unscreened long-range $1 / r$ interaction. The authors simulated systems with different persistence lengths, linear charge densities of the chain and ball radii. Depending on the choice of parameters, they encountered a multitude of structures-ranging from collapsed structures with a "tennisball seam pattern" or solenoid arrangement of the wrapped chain ${ }^{9}$ to open multileafed structures very much resembling the ones found in ref 10. Their rosette structures occur for stiffer chains on smaller spheres.

That work demonstrates that the rosette structure is quite robust with respect to the range of interaction. At the same time it al so hints toward major differences between these two cases: The short-ranged case predicts clearly a sharp unwrapping transition into largeleafed rosettes when the adsorption energy is decreased or the chain stiffness is increased. ${ }^{10}$ In the simulation, 32 however, the rosette evolved continuously with increasing chain stiffness from a tightly wrapped state via slightly more open structures with many small loops to large-leafed rosettes.

How can one reconcile these findings? For that purpose we reconsider in section 2 the chain-sphere complexation for the case of short-ranged attraction, 10 formulating now the interaction in the language of strongly screened electrostatics instead of some shortranged stickiness. Then, in section 3 , we give the scaling description in the case of weak screening. By focusing on the unwrapping transition we contrast the two cases in section 4. In the final section we compare our findings to computer simulations and experimental observations on the DNA-histone complex.

\section{Charged Rosettes at High Ionic Strength}

Consider a polymer chain of length $L$, radius $r$, and persistence length $I_{p}$. The chain carries negative charges on its backbone- $a$ distance $b$ apart-which leads to $a$ linear charge density- $\mathrm{e} / \mathrm{b}$. The macroion is modeled as a sphere of radius $\mathrm{R}$ that carries a positive charge $Z$. The reduced electrostatic potential is assumed to be smaller than unity everywhere so that the el ectrostatic interaction can be described by standard Debye-Hückel theory: Two elementary charges at distance $r$ interact via the potential $\mathrm{e} \phi / \mathrm{kT}= \pm \mathrm{I}_{\mathrm{B}} \mathrm{e}^{-\kappa \mathrm{r}} / \mathrm{r}$ with $\mathrm{I}_{\mathrm{B}}=\mathrm{e}^{2} / \epsilon \mathrm{k}_{\mathrm{B}} T$ $\left(\epsilon\right.$, dielectric constant of the solvent; $\mathrm{k}_{\mathrm{B}} \mathrm{T}$, thermal energy), the Bjerrum length, and $\kappa^{-1}=\left(\left.8 \pi\right|_{B} C_{S}\right)^{-1 / 2}$, the Debye screening length for monovalent salt of concentration $C_{5} . I_{B}$ is on the order of $7 \AA$ in water at room temperature. Note that the applicability of the standard Debye-Hückel theory is restricted to weakly charged components (with $b>I_{B}$ and $Z<R / I_{B}$ ) where nonlinear effects like counterion condensation are not present. We shortly comment on the nonlinear case in section 4.
In this section, we assume strong screening such that the screening length is much smaller than the radius of the ball, $\kappa R \gg 1$. We will assume throughout the chain to be so thin that $r$ is the smallest length scale in our system, especially that always $\kappa r \ll 1$. The adsorption energy per length can then be estimated from the Debye-Hückel electrostatic potential close to the surface of the sphere which mimics for $\kappa \mathrm{R} \gg 1$ that of a plane with a surface charge density $\sigma=Z /\left(4 \pi \mathrm{R}^{2}\right)$. One has then $\mathrm{e} \phi / \mathrm{kT}=\mathrm{I}_{\mathrm{B}} \mathrm{Z} \mathrm{e}^{-\kappa z} / 2 \kappa \mathrm{R}^{2}$ where $\mathrm{z}$ is the distance from the surface (cf., for instance, ref 8). This leads to the following adsorption energy per length (in units of $k_{B} T$ )

$$
\lambda \simeq \frac{\mathrm{I}_{\mathrm{B}} \mathrm{Z}}{\mathrm{b}_{\kappa} \mathrm{R}^{2}}
$$

Now the chain will only wrap on the sphere when $\lambda$ is large enough, namely so large that it exceeds $I_{P} / 2 R^{2}$, the bending energy per length. ${ }^{33}$ The (free) energy of the wrapped chain-sphere complex is then given by

$$
\frac{\mathrm{F}_{\text {wrap }}}{\mathrm{K}_{\mathrm{B}} \mathrm{T}} \simeq \frac{\mathrm{I}_{\mathrm{P}} \mathrm{L}}{\mathrm{R}^{2}}-\lambda \mathrm{L}=\left(\frac{\mathrm{I}_{\mathrm{P}}}{\mathrm{R}^{2}}-\lambda\right) \mathrm{L}
$$

This leads to the prediction of an unwrapping transition at $\lambda=\mathrm{I}_{\mathrm{P}} / \mathrm{R}^{2}$ when the chain stiffness

$$
\mathrm{I}_{\mathrm{P}} \simeq \frac{\mathrm{I}_{\mathrm{B}} \mathrm{Z}}{\mathrm{b} \kappa}
$$

is reached. Since the wrapping path of the chain on the sphere can be quite intricate (cf. ref 9) the local radius of curvature is not always precisely $R$ (but on the order of R). Thus, we dropped from eq 2 on all numerical prefactors and will also do so in the rest of the paper (for similar reasons). Such an unwrapping transition has been first discussed by Marky and Manning ${ }^{19}$ using some unspecified short-range attraction and by Netz and J oanny ${ }^{8}$ for the el ectrostatic case (in fact, their eq 35 coincides with eq 3 ).

For larger persistence lengths than the one given in eq 3 the chain has to completely unwrap from the sphere. This led the authors of ref 19 to the prediction of an "all-or-nothing" picture: either the chain is wrapped or it is unwrapped-with a single adsorption point or even completely desorbed. We showed, however, in a later study that the chain can lower its energy considerably by having multiple point contacts to the sphere which leads to the rosette structures. ${ }^{10}$ Let us call $\mu$ the energy per contact point (in units of $k_{B} T$ ). Its value

$$
\mu \simeq \lambda \sqrt{\mathrm{R} \kappa^{-1}} \simeq \frac{\mathrm{I}_{\mathrm{B}} \mathrm{Z}}{\mathrm{b}(\kappa \mathrm{R})^{3 / 2}}
$$

follows from the length $\sqrt{\mathrm{R}^{-1}}$ of a chain portion around a point contact that is located within the distance $\kappa^{-1}$ from the sphere. The free energy of an $\mathrm{N}$-leafed rosette has then approximately the following form

$$
\frac{\mathrm{F}_{\text {rosette }}}{\mathrm{k}_{\mathrm{B}} T} \simeq \frac{\mathrm{I}_{\mathrm{P}}}{\mathrm{L}} \mathrm{N}^{2}-\mu(\mathrm{N}+1)
$$




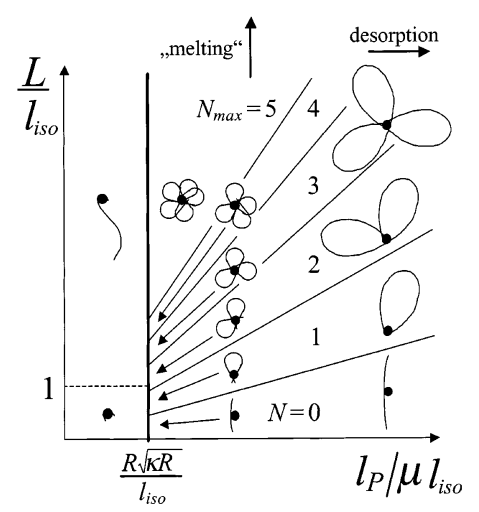

Figure 1. Sphere chain complex at high ionic strength, $\kappa \mathrm{R} \gg 1$. Depicted is the "phase" diagram as a function of the total length $L$ of the chain and of its persistence length $I_{P}$ divided by the point contact energy $\mu$ (both axes are in units of the isoelectric wrapping length $\mathrm{l}_{\text {iso }}$ ). The thick vertical bar denotes the sharp unwrapping transition. To the left are the wrapped complexes; to the right are the rosettes. Wrapped complexes show tails for sufficiently long chains (cf. main text for details).

The first term describes the energy required to bend the $\mathrm{N}$ chain pieces between the point contacts, each of length $\mathrm{L} / \mathrm{N}$, into leaves with typical curvature $\mathrm{L} / \mathrm{N}$, the second term accounts for the $\mathrm{N}+1$ point contacts. We do not include here the entropy of the chain configurations that can be safely neglected for large point contact energies $\mu \gg 1$. Note that $\mu$ can be quite large even for weakly charged components, $\mathrm{b}>\mathrm{I}_{\mathrm{B}}$ and $\mathrm{Z}<\mathrm{R} / \mathrm{I}_{\mathrm{B}}$, and strong screening, $\kappa R \gg 1-$ as assumed here. In fact, using eq 4 and assuming the sphere and chain to be just at the onset of counterion condensation, i.e., $b \approx I_{B}$ and $\mathrm{Z} \approx \mathrm{R} / \mathrm{I}_{\mathrm{B}}$ one finds a large range of $\kappa$ values which is consistent with our assumptions $\mu \gg 1$ and $\kappa \mathrm{R} \gg 1$, namely $\mathrm{I}_{\mathrm{B}}^{2 / 3} \mathrm{R}^{1 / 3} \ll \kappa^{-1} \ll \mathrm{R}$. The optimal number of leaves follows then from the minimization of the (free) energy to be

$$
\mathrm{N}^{*} \simeq \mu \frac{\mathrm{L}}{\mathrm{I}_{\mathrm{P}}}
$$

which leads to leaves of size

$$
\mathrm{I}_{\text {leaf }} \simeq \frac{\mathrm{L}}{\mathrm{N}^{*}} \simeq \frac{\mathrm{I}_{\mathrm{P}}}{\mu}
$$

As already pointed out in ref 10 each leaf minimizes its bending energy by assuming the shape of a so-called Yamakawa-Stockmayer (YS) loop, namely a lemniscate-shaped loop with an $81^{\circ}$ apex angle ${ }^{34}$ (cf. also ref 16 for an approximate derivation of the YS loop). As a cautionary remark we mention that the bending energy of YS loops leads to the numerical prefactor 14.04 for the first term in eq $5^{34}$-clearly demonstrating that one should not extract from this theory any numbers but just scaling relations. The leaves show negligible shape fluctuations for $I_{\text {leaf }} \ll I_{p}$, i.e., for $\mu \gg 1$. Then $N^{*}$, eq 6 , is essentially fixed, i.e., the rosette has a well-defined leaf number-as implicitely assumed here.

In Figure 1, we summarize our results in a "phase diagram" of total chain length $L$ vs $I_{\mathrm{p}} / \mu$ (which is in the case of rosettes nothing but the leaf size, cf. eq 7). Both axes are arbitrarily given in units of $I_{\text {iso, }}$ a choice that will turn out to be convenient for the long-ranged case discussed below. For small values of $I_{p} / \mu$ we have wrapped structures, for large values rosette conforma- tions. The sharp unwrapping transition occurs at $\mathrm{I}_{\mathrm{p}} / \mu \simeq \mathrm{R} \sqrt{\kappa \mathrm{R}}$ (cf. eqs 3 and 4 ) and is indicated by a thick vertical line. The other lines indicate transitions between different ground states, namely rosettes with different numbers of leaves.

Additional features arise from the fact that the sphere surface is finite. This can lead to the formation of tails for the case of wrapped structures and to the melting of rosettes. Consider first a wrapped chain configuration, i.e., assume some fixed value for $I_{\mathrm{P}} / \mu<\mathrm{R} \sqrt{\kappa \mathrm{R}}$, and increase $L$, i.e., go along a vertical line in the diagram, Figure 1. It is clear that at a certain point the wrapping has to stop. Locally the wrapped chain forms a nearly planar lamellar phase with a distance $d$ between neighboring chain sections. This distance follows from the competition between the chain-sphere attraction and the repulsion between neighboring chain segments $8,35,36$

$$
\mathrm{d} \simeq \frac{1}{\mathrm{~b} \sigma} \simeq \frac{\mathrm{R}^{2}}{\mathrm{bZ}}
$$

This leads to a wrapping length $\mathrm{I} \simeq \mathrm{R}^{2} / \mathrm{d} \simeq \mathrm{bZ} \simeq \mathrm{I}_{\text {iso }}$ which is just the isoelectric wrapping length, the length at which the wrapped chain portion just compensates the sphere charge. Chains that are shorter than this length, $L<I_{\text {iso, }}$ will be completely wrapped, chains that are longer, $L>I_{\text {iso, }}$, will have their extra length $L-I_{\text {iso }}$ dangling from the complex in the form of one or two tails. The tail formation at $L=I_{\text {iso }}$ has been indicated in Figure 1 by a horizontal dashed line. We hasten to note that the onset of tail formation is only taking place around this line for sufficiently tight wrapping and not too strong screening such that $I_{\text {iso }} \gg R(\kappa R) .{ }^{21}$ Otherwise the wrapping length can be much larger: If $d>\kappa^{-1}$ in eq 8, i.e., if $Z<\mathrm{R}^{2} \kappa / \mathrm{b}$ (large sphere), the lamellar spacing is reduced to the value $\kappa^{-1}$ as pointed out by Netz and J oanny. ${ }^{8}$ Then the wrapping length is on the order of $\mathrm{R}^{2} \kappa$ which is much larger than $\mathrm{I}_{\text {iso }}$ and tail formation sets in only for much longer chains. Additional effects come from the chain stiffness that in all the above cases reduce the amount of wrapping; a detailed analysis will be given elsewhere.21

Now let us consider the rosettes. Choose some arbitrary value for $I_{\mathrm{P}} / \mu>\mathrm{R} \sqrt{\kappa \mathrm{R}}$ and increase $\mathrm{L}$. Then each time when the chain length is increased by an amount $I_{\mathrm{P}} / \mu$, an additional leaf is formed. However, packing constraints imply an upper limit for the number of loops on the order of $\mathrm{N}_{\max } \approx \mathrm{I}_{\mathrm{iso}} / \sqrt{\mathrm{R}^{-1}}$; this is the maximum number of contacts, each excluding an area $\approx \mathrm{d} \sqrt{\mathrm{R}^{-1}}$, which can be closely packed on the surface of the sphere. For a rosette with the maximum number of leaves (in Figure 1 we chose arbitrarily $\mathrm{N}_{\max }=5$ ) an increase in $L$ leads to an increase of the leaf size. $I_{\text {leaf }}$ reaches the persistence length when $\mathrm{L} \simeq \mathrm{N}_{\text {max }}$ p. At that point mel ting of the rosette takes place, i.e., for larger values of $L$ the leaf size distribution is heterogeneous, the YS loops show shape fluctuations and also the tails start to grow. Entropic effects are then important. The thermodynamics of this melting process is quite intricate and can be calculated by mapping this problem on an exactly solvable one-dimensional many body problem. ${ }^{10,21} \mathrm{In}$ the current study we will not discuss the rosette melting further and merely indicate it in Figure 1 by an arrow pointing toward the direction of the $L$ axis. Also shown in this figure is the direction where 
the chain desorption is to be expected. It should occur when the complexation free energy of the rosette, eq 5 , becomes smaller than $k_{B} T$, i.e., for $I_{P} / \mu>\sqrt{I_{P} L}$.

\section{The Rosette State at Low I onic Strength}

The rosette configurations discussed in the last section occur for chains that are so stiff that wrapping would be too costly. It allows a small fraction of the chain to be close to the sphere in the form of point contacts. The majority of the monomers resides in the loops that do not "feel" the presence of the sphere but are needed to connect the point contacts via low curvature sections. At first sight, one might thus expect the rosette configurations to be a special feature for chain-sphere complexes with a short ranged mutual attraction.

That this is not true has al ready been pointed out in the Introduction. As observed in the simulations by Akinchina and Linse, ${ }^{32}$ rosettes are in fact quite robust and occur also in systems with a much larger range of interaction. We shall give now a scaling theory that provides us with a diagram of states for the chainsphere complex for the case of weak screening, $\kappa \mathrm{R} \ll 1$. We will first treat the case of short chains $L=b N \leq$ $\mathrm{bZ}=\mathrm{I}_{\text {iso }}$ where the chain charge is smaller than (or equals) the sphere charge. The free energy of the rosette with $\mathrm{N}$ leaves is then approximately given by

$$
\frac{F_{\text {rosette }}}{\mathrm{k}_{B} T} \simeq \frac{\mathrm{I}_{\mathrm{P}}}{\mathrm{L}} \mathrm{N}^{2}-\frac{\mathrm{I}_{\mathrm{B}} \mathrm{Z}}{\mathrm{b}} \mathrm{N}
$$

The first term is the bending energy of $\mathrm{N}$ leaves as al ready discussed after eq 5 . The second term accounts for the attraction between the ball charge $Z$ and the chain charge L/b over the typical distance L/N . All other contributions to the electrostatics like the monomermonomer repulsion are smaller and neglected in eq 9. Remarkably this free energy has the same form as the free energy 5 of the rosette for short-ranged interaction. We just have to identify the point contact energy $\mu$ (for the strong screening case) with the single leaf-sphere interaction

$$
\mu \simeq \frac{\mathrm{I}_{\mathrm{B}} \mathrm{Z}}{\mathrm{b}}
$$

for the unscreened case. The optimal leaf number is thus again given by eq 6 and the leaf size by eq 7 but now with $\mu$ given by eq 10 . Note that these scaling results remain even true if the leaves grow so large that their outer sections do not interact with the sphere due to screening, $\kappa l_{\text {leaf }}>1$. This is so because the el ectrostatic rosette-sphere attraction still scales like $-I_{B} Z N / b$ : a fraction $\kappa^{-1} /(\mathrm{L} / \mathrm{N})$ (i.e., $\kappa^{-1} / \mathrm{b}$ charges) of each of the $\mathrm{N}$ leaves interacts with the sphere at a typical distance $\kappa^{-1}$.

The rosette state competes with the wrapped state. We expect that the rosette state is continuously transformed into the wrapped state when $I_{\text {leaf }} \simeq R$; then, the leaves become so small that they touch with their contour the surface of the sphere. Indeed, by setting $\mathrm{N}=\mathrm{L} / \mathrm{R}$ in eq 9 , one finds

$$
\frac{F_{\text {wrap }}}{k_{B} T} \simeq \frac{I_{P} L}{R^{2}}-\frac{I_{B} Z L}{b R}=\left(\frac{I_{P}}{R^{2}}-\frac{I_{B} Z}{b R}\right) L
$$

which can be considered as the free energy of the wrapped state: The first term is the bending energy required to wrap the chain around the sphere and the second accounts for the el ectrostatic attraction between the wrapped chain and the sphere. All other electrostatic contributions (as discussed in detail by Nguyen and Shklovskii ${ }^{11}$ ) are less important and do not occur on this level of approximation. On the right-hand side of eq 11, we arranged the terms in such way that one can deduce directly an unwrapping transition, namely at $I_{P} / R^{2}=I_{B} Z / b R$ which can be rewritten as

$$
\mathrm{I}_{\mathrm{P}} \simeq \mu \mathrm{R} \simeq \frac{\mathrm{I}_{\mathrm{B}} \mathrm{ZR}}{\mathrm{b}}
$$

Comparing eqs 2 and 11 , one might expect that the chain unwraps in a strongly discontinuous fashion as discussed in the previous section. However, this "unwrapping point" corresponds just to the point $I_{\text {leaf }} \simeq R$ when small loops start to grow on the sphere; we thus expect the transition to be continuous as pointed out before eq 11. That the unwrapping transition occurs rather smooth at low ionic strength and sharp at high ionic strength has been predicted by N etz and J oanny; ${ }^{8}$ however, in that study the authors did not allow for rosette structures.

To complete the picture, we finally have to consider chains that are longer than the isoelectric length, $\mathrm{L}>\mathrm{I}_{\text {iso. }}$. We need then at least three terms to capture the essential physics of the rosette state:

$$
\frac{\mathrm{F}_{\text {rosette }}}{\mathrm{k}_{\mathrm{B}} T} \simeq \frac{\mathrm{I}_{\mathrm{P}}}{\mathrm{I}} \mathrm{N}^{2}-\frac{\mathrm{I}_{\mathrm{B}} \mathrm{Z}}{\mathrm{b}} \mathrm{N}+\frac{\mathrm{I}_{\mathrm{B}} \mathrm{I}}{\mathrm{b}^{2}} \mathrm{~N}
$$

Here we "allow" the monomers to distribute between the rosette of length I and a tail of length $L-I$. The first two terms in eq 13 are then as above, eq 9, the last term describes the repulsion of the monomers that constitute the rosette. The contributions from the tail can be neglected. Minimization with respect to I leads to the optimal rosette length

$$
\mathrm{I}^{*} \simeq \mathrm{b} \sqrt{\frac{\mathrm{I}_{\mathrm{P}} \mathrm{N}}{\mathrm{I}_{\mathrm{B}}}}
$$

The free energy, eq 13, with the optimal length I*, eq 14, is minimized for the following number of leaves:

$$
\mathrm{N}^{*}=\mu \frac{\mathrm{I}_{\text {iso }}}{\mathrm{I}_{\mathrm{P}}}
$$

Hence-on this level of approximation $-I^{*} \simeq b \sqrt{I_{\mathrm{P}} N * / I_{\mathrm{B}}}$ $\simeq I_{\text {iso; }}$ i.e., the rosette monomers just compensate the central ball charge; the rest of the monomers extend away from the rosette in one or two tails of total length $L-I_{\text {iso. }}$ Each leaf is of size

$$
\mathrm{I}_{\text {leaf }} \simeq \frac{\mathrm{I}}{\mu}
$$

The rosette disappears at $I_{\text {iso }} / N^{*}=R$, i.e., when eq 12 is fulfilled. It is then replaced by a wrapped structure of length $I_{\text {iso }}$ and one or two tails having the total length $L-I_{\text {iso. }}$

In Figure 2, we depict the complete diagram of the sphere-stiff chain complexes to be expected in the unscreened case. We again plot $\mathrm{L}$ vs $I_{\mathrm{p}} / \mu$, the leaf sizeboth in units of $I_{\text {iso. }}$. When one starts in this diagram at 


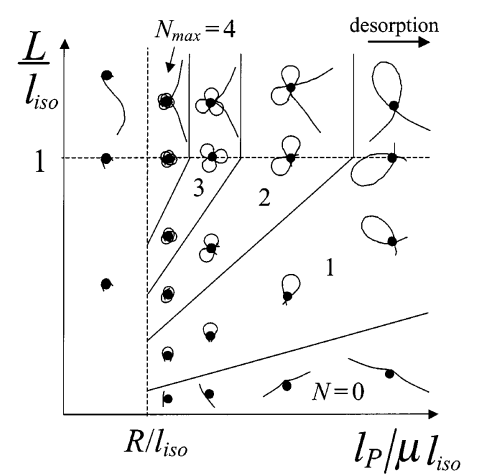

Figure 2. Diagram of states for the case of low ionic strength, $\kappa \mathrm{R} \ll 1$. The axes are chosen similar to Figure 1 with $\mu$ now being the leaf-sphere attraction in the rosette structures. The unwrapping is here rather smooth (dashed vertical line). Complexes with long chains, $L>I_{\text {iso, }}$ show tails.

(a)

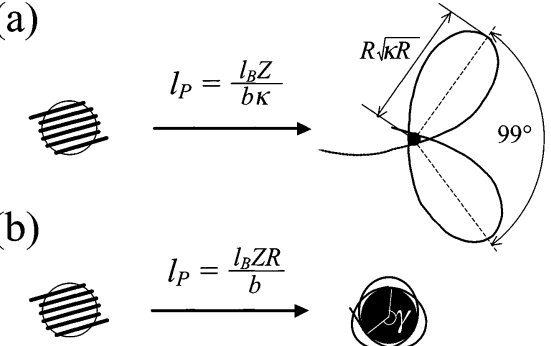

Figure 3. Unwrapping transition (a) at high ionic strength, $\kappa \mathrm{R} \gg 1$ and (b) at low ionic strength, $\kappa \mathrm{R} \ll 1$. In the former case, the transition is sharp and large Yamakawa-Stockmayer loops are formed; in the latter case, the rosette structures evolves continuously.

a large value of $\mathrm{I}_{\mathrm{p}} / \mu$ and goes toward smaller values of (with some arbitrarily fixed value $L<I_{\text {iso }}$ ) then all leaves shrink and more and more leaves can form. At $I_{\mathrm{p}} / \mu=\mathrm{R}$ the maximum number of leaves (for that given value of $L$ ) is reached, and at the same time the leaves di sappear simultaneously in a continuous fashion. For $\mathrm{I}_{\mathrm{p}} / \mu<\mathrm{R}$ the chain wraps around the sphere. For long chains, $L>I_{\text {iso, }}$ the excess charges are accommodated in tails and all rosettes have the same length $I_{\text {iso. }}$. The borderlines between different rosette ground states are then independent of the total length of the chain and thus appear in Figure 2 as vertical lines. Wrapped complexes with long chains $L>I_{\text {iso }}$ al so show tails of length $L-$ $\mathrm{I}_{\text {iso; }}$ correction terms on the order of R (small compared to $\mathrm{I}_{\text {iso }}$ for multiple turns) have been extensively discussed in the literature (cf., for instance, ref 11 ).

Desorption occurs when the free energies, eqs 9 and 13, equal the thermal energy $k_{B} T$. This point is reached when $I_{\mathrm{P}} / \mu \simeq \sqrt{I_{\mathrm{P}} \mathrm{L}}$ for short chain, $\mathrm{L} \leq \mathrm{I}_{\text {iso, }}$ and when $I_{\mathrm{p}} / \mu \simeq \sqrt{I_{\mathrm{p}} I_{\text {iso }}}$ for long chains, $L>\mathrm{I}_{\text {iso. }}$. We indicate in Figure 2 the direction where desorption occurs by an arrow.

\section{Unwrapping at High and Low Ionic Strength}

We take now a closer look at the unwrapping transition, contrasting the short-range case and the longrange case. The former case, $\kappa \mathrm{R} \gg 1$, is depicted in Figure 3a. As discussed in section 2, we expect the unwrapping transition to occur at $\lambda \simeq \mathrm{I}_{\mathrm{p}} / \mathrm{R}^{2}$ which leads to eq 3. At this point the structure jumps in a dramatic fashion into a largeleafed rosette with leaves of size $I_{\mathrm{P}} / \mu$; cf. eq 7. Combining eqs 3,4 , and 7 , we find indeed

$$
\mathrm{I}_{\text {leaf }} \simeq \mathrm{R} \sqrt{\kappa \mathrm{R}} \gg \mathrm{R}
$$

As discussed in section 2 each leaf has the shape of a YS loop ${ }^{34}$ with an $81^{\circ}$ apex angle. This means that neighboring leaves have a relative orientation $\theta=180^{\circ}$ $-81^{\circ}=99^{\circ}$, of. Figure 3a. In addition, the leaves have to be slightly twisted (like propeller blades) to account for the mutual excluded volume.

The unwrapping at low ionic strength is depicted in Figure $3 \mathrm{~b}$ and goes as follows (we discuss here the case $\mathrm{L} \leq \mathrm{I}_{\text {iso; }}$ in the opposite case, one has just to replace $\mathrm{L}$ by $\left.I_{\text {iso }}\right)$. When the chain becomes so stiff that $I_{P} / R^{2}>$ $\mathrm{I}_{\mathrm{B}} \mathrm{Z} /(\mathrm{bR})$ the wrapped state is not stable anymore (cf. eq 11). At that point many small leaves ( $\mathrm{N}^{*}=\mathrm{L} / \mathrm{R}$ ones) form simultaneously in a continuous fashion. Their size scales as $\mathrm{I}_{\text {leaf }} \simeq \mathrm{R}$, the precise prefactor being not accessible to our scaling argument. The number of windings around the ball scales as L/R (but note that the geometry of the wrapped path can be actually quite intricate as demonstrated in ref 9) and the typical opening angle $\gamma$ of each loop at the point of its formation scales as $(\mathrm{L} / \mathrm{R}) / \mathrm{N}^{*} \approx 1$, again with an unknown numerical value. A multileafed configuration slightly above the unwrapping point is depicted schematically on the righthand side of Figure 3b.

Additional insight can be gained by generalizing the attractive force between a given chain charge and the sphere by a power law $-\mathrm{AZ} / \mathrm{r}^{\alpha}$ with an arbitrary exponent $\alpha>0$. An integer value $\alpha=D-2$ with $D=$ $3,4, \ldots$, can be interpreted as a charged chain that adsorbs on an oppositely charged D-dimensional ball in $\mathrm{D}$ dimensions. The electrostatic term for the rosette in eq 9 takes then the form $-\mathrm{AZN} \alpha /\left(\mathrm{bL}^{\alpha-1}\right)$ and the one for the wrapped state scales as $-\mathrm{AZL} / \mathrm{bR}^{\alpha}$. Unwrapping takes place at $I_{p} * \simeq A Z /\left(b^{\alpha-2}\right)$. At this critical value the free energy of the rosette $F_{\text {rosette }}\left(I_{P}=I_{P^{*}}\right.$ ) has (as a function of $N$ ) a minimum at $N^{*} \simeq L / R$ for $\alpha<2$ (D < 4), suggesting a rather smooth unwrapping transition similar to the one depicted in Figure 3b. This mi nimum turns into a maximum at $\alpha=2(D=4)$. For larger values of $\alpha$ we find $N^{*}=0$; i.e., the unwrapping transition is sharp, similar to the short-ranged case discussed in the previous section.

Finally, we note that in the case of very highly charged chains and spheres the Debye-Hückel approximation breaks down and nonlinear phenomenons like counterion condensation become important. 37,38 The dominant contribution to the complexation energy is then the release of counterions that were condensed on the sphere and on the chain before complexation. This is a short-ranged interaction and consequently the unwrapping transition is expected to be sharp-even at low ionic strength. Following ref 14, the free energy of the wrapped state can be written as

$$
\frac{\mathrm{F}_{\text {wrap }}}{\mathrm{k}_{\mathrm{B}} \mathrm{T}} \simeq\left(\frac{\mathrm{I}_{\mathrm{P}}}{\mathrm{R}^{2}}-\frac{\Omega}{\mathrm{b}}\right) \mathrm{L}
$$

where $\Omega$ is the free energy gain (in units of $k_{B} T$ ) per adsorbed chain charge which follows from the entropy gain due to counterion release ( $\Omega$ is a number of order one; for details cf. ref 14). Unwrapping into the rosette takes place at $I_{p} \simeq \Omega R^{2} / b$. The point contact energy is on the order of $(\Omega / b) \sqrt{R \lambda_{G C}}$ where the so-called GouyChapman length $\lambda_{\mathrm{GC}} \simeq 1 /\left(\sigma \mathrm{I}_{\mathrm{B}}\right)$ is the thickness of the layer of condensed counterions around the sphere (which is always much smaller than $\mathrm{R}$ when there is 
strong counterion condensation on the sphere, i.e., for $Z \gg R / I_{B}$ ). The leaf size at the unwrapping point is then given by

$$
\mathrm{I}_{\text {leaf }} \simeq \sqrt{\frac{\mathrm{R}}{\lambda_{\mathrm{GC}}}} \mathrm{R} \gg \mathrm{R}
$$

which indeed indicates a sharp unwrapping transition.

\section{Discussion and Conclusion}

We compare now our results with Monte Carlo simulations by Akinchina and Linse ${ }^{32}$ and then with the behavior of a biological chain-macroion complex, the nucleosome. ${ }^{1}$ The simulated systems ${ }^{32}$ were always at the isoelectric point, i.e., the chain length was given by $\mathrm{L}=\mathrm{bZ}=\mathrm{I}_{\text {iso. }}$. Furthermore, the charges in the simulation interacted via a nonscreened Colombic $1 / r$ potential. The simulation results have thus to be compared with Figure 2. Four systems have been considered, each having having a fixed set of parameters $b, Z$, and $R$ but with seven different values of $I_{p}$. This means that for each case the systems were located on the dashed horizontal line at $\mathrm{L} / \mathrm{I}_{\text {iso }}=1$ in Figure 2 .

In one system (called system II in ref 32) the continuous development from a wrapped to the rosette configurations has been seen most clearly. Example configurations are shown in Figure 1, system II in that paper (that these are representative can be seen by inspecting the adsorption probability of monomers as a function of the monomer index, of. Figure 3 in 32 ). $F$ or $I_{p}=7 \AA$, the chain is wrapped; at $I_{P}=60 \AA$, there is al ready a slight indication of very small loops ( $\mathrm{N}=4$ or 5 , cf. the small oscillations in Figure 3, systems II, open squares). The next system depicted has al ready a much stiffer chain, $I_{P}=250 \AA$, and shows very clearly three leaves, then two leaves at $I_{p}=500 \AA$, and one leaf for the stiffest chain, $I_{p}=1000 \AA$. In Figure 2, we have chosen the parameters such that $N_{\max } \simeq I_{\text {iso }} / R$ equals 4 so that this corresponds roughly to system II in 32. To compare with the simulations we have to follow the $L=I_{\text {iso-line }}$ in Figure 2: One starts with wrapped structures for $\mathrm{I}_{\mathrm{P}} / \mu<\mathrm{R}$ and finds then the continuous evolution of rosettes when the line $I_{\mathrm{p}} / \mu=\mathrm{R}$ is crossed. The leaves grow at the expense of their number (first four, then three, and two leaves), just as it has been observed in the simulations.

In another system (system I), all parameters were kept the same except the sphere radius that was now twice as large. This system shows three small loops when $I_{p}=250 \AA$ is reached and one loops for $I_{p}=500$ and $1000 \AA$. In our "phase diagram" the transformation $\mathrm{R} \rightarrow 2 \mathrm{R}$ means that the vertical unwrapping transition line $\left(I_{\mathrm{P}} / \mu=\mathrm{R}\right)$ moves to the right and consequently the maximum number of loops goes down by a factor of 2 : $\mathrm{N}_{\max } \rightarrow \mathrm{N}_{\max } / 2$ which is in satisfactory agreement with the finding in 32. Going from our reference system (II) to another case (system IV) means to go to a higher line charge density of the chain, i.e., the transformation $b \rightarrow b / 2$ (and thus $I_{\text {iso }} \rightarrow I_{\text {iso }} / 2$ ). In that case the unwrapping transition line moves again to a larger value leading to $\mathrm{N}_{\max } \rightarrow \mathrm{N}_{\max } / 2$. The complex in ref 32 shows an unwrapping only for $I_{p}>500 \AA$ and has al ready a well-pronounced loop at $I_{p}=1000 \AA$. Apparently, the wrapped state is more stable in this system than in system I - and the scaling argument predicts indeed the critical persistence length to scale as $1 / b$, cf. eq 12 . The last system (III) finally combines a large sphere, $R \rightarrow 2 R$, with a short chain, $b \rightarrow b / 2$. In that case no indication of loops has been observed. Even at the largest persistence length the chain is touching the sphere over some finite section (similarly to the geometry discussed by N etz and J oanny ${ }^{8}$ ). I $n$ fact, we predict $\mathrm{N}_{\max } \rightarrow \mathrm{N}_{\text {max }} / 4$ by going from the reference system (II) to this case. Altogether, our scaling approach is in quite satisfactory agreement with the observations made in the Monte Carlo simulations. ${ }^{32}$ It would be interesting to test our predictions also for chains that are shorter or longer than the isoelectric length. Corresponding simulations are already on the way. ${ }^{39}$

Now we compare our scaling results with an experimental system, the nucleosome. ${ }^{1}$ The DNA in the nuclei of plant and animal cells forms together with so-called histone proteins the dense chromatin complex. The basic step is the wrapping of DNA around protein spools, each made from eight histones. The repeating unit of this complex, wrapped DNA, histone octamer and a stretch of linker DNA (connecting to the next such protein spool) is called nucleosome. When the linker is digested away the remaining complex, the so-called nucleosome core particle, consists of 147 basepairs $(\approx 500 \AA)$ DNA wrapped in $13 / 4$ left-handed superhelical turns around the octamer. The core particle which has a radius of $\sim 50$ $\AA$ and a height of $\sim 60 \AA$ is documented in great detail on the basis of high-resolution X-ray analyses. ${ }^{40}$ The histone octamer contains 220 basic side chains (arginine and lysine). ${ }^{41}$ From these are about 103 located in flexible histone tails that dangle of the core particle and/ or are complexed with the wrapped DNA.42 The rest, 117 residues, are in the globular part of the octamer, of which 31 are exposed to the solvent, the rest being involved in intra- and interprotein ionic interactions. On the other hand, one has 147 bps of DNA wrapped around the octamer, each contributing two phosphate groups. Hence there are 294 negative charges from the DNA vs 220 positive charges of the octamer, i.e., the nucleosomal complex is overcharged by the DNA.

Yager, McMurray, and van $\mathrm{Holde}^{43}$ characterized the stability of the nucleosome core particle as a function of the salt concentration $(\mathrm{NaCl})$. Using a variety of experimental methods (e.g., gel electrophoresis) they arrived at the following main conclusions: The core particle is stable for ionic strengths ranging from $2 \mathrm{mM}$ to $750 \mathrm{mM}$ (this includes physiological relevant salt concentrations around $100 \mathrm{mM}$ ). For slighly higher salt concentrations the DNA is partially dissociated; an equilibrium between histones, free DNA and core particles is observed. At salt concentrations beyond $1.5 \mathrm{M}$ the core particle is completely dissociated into histone oligomers and free DNA. On the other end, for very low salt concentration $\leqslant 1 \mathrm{mM}$ one finds an "expanded" state of the nucleosome. Khrapunov et al ${ }^{41}$ came via fluorescence measurements to similar conclusions: For ionic strengths between 5 and $600 \mathrm{mM}$ the core particle is intact. At larger ionic strength $(\approx 1.2 \mathrm{M})$ the terminal regions of the DNA unwrap and two histone dimers are dissociated and at a even larger value $(\approx 1.5 \mathrm{M})$ the remaining tetramer leaves the DNA. Finally, at low salt concentrations one encounters an open state: the dimers break their contact with the tetramer and the DNA termini unwrap.

The key features of the behavior of core particle DNA (neglecting the substructure of the octamer) were re covered in a numerical study by Kunze and N etz. ${ }^{9}$ They considered the complexation of a charged, semiflexible 
chain with an oppositely charged sphere, interacting via a screened Debye-Hückel potential. The optimal DNA configuration was derived numerically from the minimization of the energy. For a reasonable set of parameters (comparable to the one of the core particle) they found for vanishing ionic strength $\left(\kappa^{-1} \rightarrow \infty\right)$ an open, planar configuration where only a small fraction of the chain is wrapped whereas the two tails (of equal length) are extended into roughly opposite directions. This is reminiscent of the open structures reported in the experimental studies. ${ }^{41,43}$ Upon addition of salt the structure goes from a two- to a one-tail configuration and the chain begins to wrap more and more. Already well below physiological ionic strengths the chain is almost completely wrapped. The chain stays in this wrapped state up to very high salt concentrations. Only then does the chain unwrap in a sharp fashion when the chain-sphere attraction is sufficiently screened. Also these features of the complex (a wrapped compact state in a wide range around physiological conditions and unwrapping at high salt content) reflect the experimental finding in refs 41 and 43.

How are these findings related to our scaling study? Let us start at physiological salt concentrations around $100 \mathrm{mM}$. Then $\kappa^{-1} \simeq 10 \AA$ is much smaller than the particle size $\approx 100 \AA$ and we have the short-range case. Since $r \simeq 10 \AA$, i.e., $k r \approx 1$ for physiological conditions, and since the binding sites between DNA and the histones are quite specific, ${ }^{40}$ the estimation of $\lambda$ in eq 1 is not reliable. $\lambda$ can be derived instead experimentally from competitive protein binding to nucleosomal DNA 44 to be on the order of $(1 / 5) \mathrm{k}_{B} T / \AA \AA$ which is roughly 10 times smaller than what eq 1 would predict (but note also that contact is made mainly between DNA minor grooves which are 10 basepairs apart!). This short-range attraction is largely balanced by the strong bending contribution $\left(I_{P}=500 \AA\right.$ for DNA and hence $I_{P} /\left(2 R^{2}\right) \simeq 1 / 6-$ $\left.1 / 7 \mathrm{k}_{\mathrm{B}} \mathrm{T} / \AA \mathrm{A}\right)$. In any case, eq 3 predicts an unwrapping for sufficiently small values of $\kappa^{-1}$ but the numbers are not reliable. The more interesting case is that of the core particle at low ionic strength around $1 \mathrm{mM}\left(\kappa^{-1} \simeq 100\right.$ $\AA)$. At that salt concentration $2 \kappa \mathrm{R} \approx 1$ and we enter the long-range case. Apparently, the completely wrapped configuration is not stable at this point anymore. This is not surprising since the nucleosomal DNA overcharges the protein octamer by at least 74 negative charges (if not by $\approx 160$ charges since 86 charged residues are buried inside the octamer). We thus expect a considerable part of the terminal DNA to unwrap and to be part of one or two tails. In Figure 2, this corresponds to the wrapped chain structures with tail that are found for small values of $\mathrm{I}_{\mathrm{P}} / \mu, \mathrm{I}_{\mathrm{p}} / \mu<\mathrm{R}$, and large values of $L, L>I_{\text {iso. }}$.

It would be interesting to redo the experiments with DNA pieces that are longer than 147 basepairs. For sufficiently large salt concentrations there might be then the occurrence of rosettes (if there is no interference with the partial disintegration of the octamer). For the other limit, it might be appropriate to use the argument for highly charged chains and spheres as given at the end of the previous section. The linear charge density of DNA is very high (two phosphate groups per base pair, i.e., per $3.4 \AA$ ). Manning theory ${ }^{38}$ indeed predicts that counterion condensation reduces the linear charge density to $-e \mathrm{I}_{\mathrm{B}}$ with $\mathrm{I}_{\mathrm{B}}=7 \AA$. Also the charge of the histone octamer is so high that counterion condensation is important. For that case, we predicted above the unwrapping to take place around $I_{\mathrm{P}}=\Omega \mathrm{R}^{2} / \mathrm{b}$. However, since the DNA persistence length is smaller than $R^{2} / b \approx(50 \AA)^{2} / 1.7 \AA \approx 1500 \AA$, we expect the wrapped state to be stable at low ionic strength, and no rosettes should occur in this limit.

Concluding, we have presented a scaling theory for the complexation between a charged chain and an oppositely charged sphere for the two limits of high and low ionic strength. For strong screening one encounters with increasing chain stiffness a sharp transition from a wrapped to an open multileafed rosette structure as al ready predicted in ref 10 . In the case of weak screening one has again wrapped structures for sufficiently flexible chains and rosettes for stiffer chains. The unwrapping transition, however, is rather smooth in this case. That this is a characteristic of a system with longranged interaction has been further supported by extending this theory to power law attraction with arbitrary exponents and to the nonlinear limit of highly charged systems. We hope that the presented "phase diagrams" for the two cases are helpful for further theoretical and experimental studies.

Acknowledgment. I am grateful to Anna Akinchina and Per Linse for sending their paper, ref 32, prior to publication, and for helpful comments.

\section{References and Notes}

(1) Kornberg, R. D.; Lorch, Y. Cell 1999, 98, 285. Widom, J . Annu. Rev. Biophys. Biomol. Struct. 1998, 27, 285.

(2) Ganachaud, F.; Elaïssari, A.; Pichot, F.; Laayoun, A.; Cros, P. Langmuir, 1997, 13, 701.

(3) Sukhorukov, G. B.; Donath, E.; Davis, S.; Lichtenfeld, H.; Caruso, F.; Popov, Vi. I.; M öhwald, H. Polym. Adv. Technol. 1998, 9, 759.

(4) McQuigg, D. W.; Kaplan, J . I.; Dubin, P. L. J . Phys. Chem. 1992, 96, 1973 and references therein.

(5) Park, S. Y.; Bruinsma, R. F.; Gelbart, W. M. Europhys. Lett. 1999, 46, 454.

(6) Mateescu, E. M.; J eppesen, C.; Pincus, P. Europhys. Lett. 1999, 46, 493.

(7) Gurovitch, E.; Sens, P. Phys. Rev. Lett. 1999, 82, 339.

(8) Netz, R. R.; J oanny, J .-F. Macromolecules 1999, 32, 9026.

(9) Kunze, K.-K.; Netz, R. R. Phys. Rev. Lett. 2000, 85, 4389.

(10) Schiessel, H.; Rudnick, J .; Bruinsma, R.; Gelbart, W. M. Europhys. Lett. 2000, 51, 237.

(11) N guyen, T. T.; Shklovskii, B. I. Physica A 2001, 293, 324.

(12) Nguyen, T. T.; Shklovskii, B. I. J . Chem. Phys. 2001, 114, 5905.

(13) Nguyen, T. T.; Shklovskii, B. I. J . Chem. Phys. 2001, 115, 7298.

(14) Schiessel, H.; Bruinsma, R. F.; Gelbart, W. M. J . Chem. Phys. 2001, 115, 7245.

(15) Schiessel, H.; Widom, J .; Bruinsma, R. F.; Gelbart, W. M. Phys. Rev. Lett. 2001, 86, 4414; Phys. Rev. Lett. 2002, 88, 129902.

(16) Kulić, I.; Schiessel, H. Biophys. J ., in press.

(17) Odijk, T. Macromolecules 1980, 13, 1542.

(18) Pincus, P. A.; Sandroff, C. J .; Witten, T. A. J . Phys. (Paris) 1984, 45, 725

(19) Marky, N. L.; Manning, G. S. Biopolymers 1991, 31, 1543.

(20) von Goeler, F.; Muthukumar, M. J. Chem. Phys. 1994, 100, 7796.

(21) Schiessel, H. J . Phys.: Condens. Matter, in press.

(22) Wallin, T.; Linse, P. Langmuir 1996, 12, 305.

(23) Wallin, T.; Linse, P. J . Phys. Chem. 1996, 100, 17873.

(24) Wallin, T.; Linse, P. J . Phys. Chem. B 1997, 101, 5506.

(25) Wallin, T.; Linse, P. J . Chem. Phys. 1998, 109, 5089.

(26) Haronska, P.; Vilgis, T. A.; Grottenmüller, R.; Schmidt, M. Macrom. Theory Simul. 1998, 7, 241.

(27) Chodanowski, P.; Stoll, S. Macromolecules 2001, 34, 2320.

(28) J onsson, M.; Linse, P. J . Chem. Phys. 2001, 115, 3406.

(29) J onsson, M.; Linse, P. J . Chem. Phys. 2001, 115, 10975.

(30) Messina, R.; Holm, C.; Kremer, K. Phys. Rev. E 2001, 65, 041805. 
(31) Messina, R.; Holm, C.; Kremer, K. J . Chem. Phys. 2002, 117, 2947.

(32) Akinchina, A.; Linse, P. Macromolecules 2002, 35, 5183.

(33) Harries, R. A.; Hearst, j . E. J . Chem. Phys. 1966, 44, 2595.

(34) Yamakawa, H.; Stockmayer, W. J . Chem. Phys. 1972, 57, 2843.

(35) Netz, R. R.; J oanny, J .-F. Macromol ecules 1999, 32, 9013.

(36) Schiessel, H.; Aranda-Espinoza, H. Eur. Phys. J . E 2001, 5, 499.

(37) Oosawa, F. Polyel ectrolytes; Decker: New York, 1971.

(38) Manning, G. S. Q. Rev. Biophys. 1978, 11, 179.

(39) Akinchina, A. Private communication.
(40) Luger, K.; Mäder, A. W.; Richmond, R. K.; Sargent, D. F.; Richmond, T. J . Nature, 1997, 389, 251.

(41) Khrapunov, S. N.; Dragan, A. I.; Sivolob, A. V.; Zagariya, A. M. Biochim. Biophys. Acta 1997, 1351, 213.

(42) Mangenot, S.; Raspaud, E.; Tribet, C.; Belloni, L.; Livolant, F. Eur. Phys. J . E 2002, 7, 221.

(43) Yager, T. D.; McMurray, C. T.; van Holde, K. E. Biochemistry 1989, 28, 2271.

(44) Polach, K.J .; Widom, J. J . Mol. Biol. 1995, 254, 130; 1996 $258,800$.

MA0213910 